\title{
Música na era do streaming: curadoria e descoberta musical no Spotify
}

\section{Pedro Henrique Moschetta* Jorge Vieira*}

\section{Resumo}

Serviços de streaming facilitam o consumo e a descoberta de músicas gravadas ao oferecer acesso irrestrito e sob demanda a uma gigantesca coleção musical a partir de qualquer dispositivo online, em qualquer hora ou local. A curadoria, feita por humanos e/ou máquinas, tem o papel de filtrar, selecionar e guiar a experiência de consumo, sendo uma forma de lidar com a abundância e superacessibilidade de conteúdo. Após pesquisa realizada com 20 usuários do Spotify, combinando entrevistas e análise de dados, os resultados mostram que a facilidade de acesso incentiva a descoberta de novas músicas, tornando a experiência de consumo mais diversificada e fragmentada, e demonstram o papel fundamental das playlists como principal forma de descoberta e coleção musical. O estudo revela ainda que a curadoria algorítmica, apesar de ser constantemente aprimorada, não substitui a curadoria humana devido à sua maior previsibilidade e imprecisão.

Palavras-chave: Consumo, Música, Streaming, Curadoria, Descoberta.

* Instituto Universitário de Lisboa, Lisboa, Portugal. 


\section{Music in the streaming era: music curatorship and discovery in Spotify}

\section{Abstract}

Streaming boosts music consumption and discovery by offering on-demand access to a large music collection from any connected device, anytime and anywhere. Music curation - which can be done by humans or machines - is as a way to filter, select and guide consumer experience, helping users to deal with the abundance and over-accessibility of content. Results of in-depth interviews conducted with 20 Spotify users in Brazil, articulated with data collected from their digital listening practices, shows how ease of access encourages practices of music discovery and promotes a more diversified and fragmented consumer experience. Playlists are often used as mechanisms of musical discovery, collection and identity formation, being one of the main changes fostered by streaming. This study also demonstrates that algorithmic curation, although constantly improved, still does not replace human curation mainly due to its greater predictability and lack of precision.

Keywords: Consumption, Music, Streaming, Curatorship, Discovery.

\section{Introdução - streaming e o Spotify}

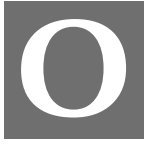

streaming é uma forma de distribuição digital que dá acesso online a um catálogo "ilimitado" de músicas gravadas, instantaneamente, em qualquer hora e local. Ao contrário de redes peer-to-peer, não exige o download antecipado das músicas, que são armazenadas em um servidor remoto e acessadas sob demanda a partir de qualquer dispositivo ligado à rede. $\mathrm{O}$ consumidor passa a ter acesso temporário às músicas, e não mais a posse - o chamado paradigma pós-posse (Sinclair; Tinson, 2017). A reprodução da música gravada, portanto, deixa de ser um bem e transforma-se em um serviço, migrando para uma "era em que o acesso é mais valioso do que a propriedade" (Hagen, 2015, p. 628). As empresas detêm controle sobre todo o conteúdo, além de um grande volume de informações sobre hábitos de consumo dos utilizadores (Fuchs, 
2017). Esses dados alimentam algoritmos ${ }^{1}$, relevantes, mas ocultos do público (Gillespie, 2014), que são utilizados, entre outras funções, para oferecer uma experiência mais personalizada aos ouvintes. A lógica do streaming é, portanto, uma "metáfora para o fluxo de informações na era digital" (Morris; Powers, 2015, p. 107), caracterizada pela abundância e superacessibilidade da informação.

Com 140 milhões de usuários, o Spotify é o serviço de subscrição (Galuszka, 2015; Fleischer, 2017) de streaming musical mais popular do mundo² (Wikström, 2013; Fleischer; Snickars, 2017), oferecendo mais de 30 milhões de músicas e 2 bilhões de playlists, sendo como tal pertinente o seu estudo sociológico.

De acordo com Hesmondhalgh (2013) são muitas as indústrias culturais que, por operarem na internet, detêm um portfólio diverso e estão ligadas ao ramo da tecnologia, mas operam igualmente com conteúdos simbólicos que são de difícil catalogação. Determinar o Spotify como objeto de estudo levanta dois desafios. O primeiro deles é mapear seus raios de ação como agregador e distribuidor de músicas. O segundo é defini-lo: será uma plataforma de conteúdos, um serviço de distribuição, ou uma companhia de mídia (Fleischer; Snickars, 2017, p. 113)?

Em termos de localização, é uma empresa sueca sediada em Estocolmo, mas está disponível atualmente em 61 países. Num breve exercício histórico, é possível retraçar a sua origem a 2006. Surgiu como uma solução tecnológica para a distribuição de conteúdos baseados numa plataforma peer-to-peer (Fleischer; Snickars, 2017). Emergiu com um catálogo, inicialmente, de música pirateada (Schwarz, 2014) e com um modelo de negócio baseado na publicidade - a necessidade de introdução, em paralelo, de um modelo de subscrição surgiu apenas mais tarde (Fleischer; Snickars, 2017). O lançamento no Brasil ocorreu em maio de 2014, após meses de testes com um grupo limitado de utilizadores.

\footnotetext{
${ }^{1}$ Algoritmos podem ser definidos como conjuntos de instruções de etapas estruturadas para processar dados com vista a produzir um output (Kitchin, 2017, p. 14).

${ }^{2}$ Dados oficiais de julho de 2017: < https://press.spotify.com/us/about/ >
} 
Após análise bibliográfica e de notícias, Fleischer e Snickars (2017, p. 138-9) defendem que o Spotify e a sua abordagem à descoberta musical passaram por três fases: a primeira delas, caracterizada pela procura individualizada, apelando à proatividade de cada consumidor, passando para uma componente social iniciada em 2010-11, e de 2013 à atualidade dando prioridade à curadoria humana e algorítmica.

Mais do que nunca, há uma crescente "necessidade de auxiliar as pessoas a descobrir, recomendar, personalizar e filtrar a quantidade enorme de conteúdo musical" (Celma, 2008, p. 12). A curadoria é, neste caso, a principal forma de selecionar, organizar e apresentar músicas, construindo significados a partir de um recorte que o curador - seja ele humano e/ou máquina - julga ser relevante para o ouvinte (Rosenbaum, 2011; Corrêa; Bertocchi, 2012). Entretanto, a previsibilidade e imparcialidade das recomendações são alguns dos efeitos colaterais apontados pela literatura.

Utilizando métodos mistos de pesquisa (Bryman, 2012), este estudo tem como objetivo discutir: a) como a facilidade de acesso pode favorecer o consumo mais variado e incentivar a descoberta de músicas; b) como os mecanismos de curadoria, apesar de falhos, impulsionam o consumo de itens menos conhecidos; e c) como playlists se tornaram uma das principais formas de consumo musical no Spotify.

\section{Evolução das formas de consumo musical}

A trajetória da música, ao longo do século XX, permite perceber como esta se tornou uma mercadoria, sendo produzida e comercializada em massa, distribuída em diversos formatos, até chegar ao que é hoje, com o streaming. Até o fim do século XIX, o consumo musical era restrito a espaços e horários limitados, como clubes, igrejas e salas de concerto (Gomes et al., 2015) e, necessariamente, com a copresença dos músicos. Com a criação do fonógrafo e do gramofone, a música passou a ser gravada e reproduzida mecanicamente, possibilitando a disjunção entre o espaço-tempo em que é produzida e consumida. Mais tarde, com as primeiras transmissões do 
rádio, na década de 1920, a música passou a ser reproduzida dentro de casa, favorecendo a emergência dos primeiros gêneros da música popular, como jazz, blues e rock.

Na década de 1940, a introdução do disco de vinil marcou o início da indústria fonográfica, responsável pela gravação, promoção e distribuição da música. Criou-se, então, uma nova lógica de mercado, em que músicas eram comercializadas na forma de singles e álbuns, com título, capa e encarte - padrão que persiste até hoje. A criação da fita cassete, na década de 1960, tornou possível a gravação doméstica das músicas, porém foi só na década de 1980 que o formato ganhou popularidade com o lançamento dos walkmans (Du Gay et al., 1997), e dos primeiros aparelhos portáteis. O surgimento do CD, no início da década de 1980, foi o marco inicial da digitalização da música, permitindo melhorias na qualidade de som a um custo mais baixo e favorecendo o crescimento exponencial da indústria fonográfica.

A maior mudança, no entanto, ocorre na década de 1990, com a introdução da internet e da compressão acústica. O formato MP3 popularizou-se graças às redes $\mathrm{P} 2 \mathrm{P}$ (peer-to-peer), eliminando a necessidade de uma mídia física e facilitando a distribuição informal de músicas (Allen-Robertson, 2013; Wikström, 2013; Schwarz, 2014). O Napster, lançado em 1999 e encerrado após dois anos, devido a uma ação judicial, talvez seja o exemplo mais conhecido. Nos anos subsequentes, a internet de banda larga e o barateamento das mídias de armazenamento favoreceram a criação de enormes coleções musicais, que mais tarde poderiam ser reproduzidas em aparelhos portáteis, como o iPod, lançado em 2001.

Mesmo com as lojas digitais, a indústria fonográfica não conseguiu impedir o aumento da distribuição informal (Lobato; Thomas, 2015), resultando em uma enorme queda nas vendas. Na metade da década de 2000, uma em cada cinco pessoas fazia o download ilegal de músicas através de ferramentas como Kazaa, LimeWire e eMule, segundo dados da 
NPD Group 3 . O mercado fonográfico passou por um longo declínio, antes de voltar a registrar, em 2016, o seu primeiro crescimento em mais de duas décadas, graças à popularização dos serviços de streaming, como o Spotify ${ }^{4}$.

\section{A relevância do estudo do consumo de música}

O estudo do consumo de música é importante para a sociologia pois "contém e reflete a linguagem, valores, sentimentos, preocupações e objetivos de uma pessoa" (Kotarba; Vannini, 2009, p. i), além de ajustar a relação entre o indivíduo e o ambiente cultural ao seu redor. Para Tia DeNora (2000, p. 7), a música é um "veículo cultural", que transporta a pessoa de um estado emocional a outro. Possui um papel importante na definição de situações cotidianas, pois "está frequentemente relacionada, através de convenções, a cenários sociais, geralmente de acordo com o uso social ao qual foi inicialmente produzida" (DeNora, 2000, p. 11). Por isso, influencia a forma como indivíduos "se conduzem, como experimentam a passagem do tempo e como se sentem [...] com relação a si mesmos, aos outros e às situações" (DeNora, 2000, p. 17). Mesma posição defende Kennedy: "As pessoas usam música diariamente para se inserirem em um determinado contexto, sociedade ou estado de espírito" (2015, p. 7).

O consumo da música também é uma ferramenta de socialização, sendo uma experiência individual e coletiva ao mesmo tempo. Individual, pois reflete gostos e preferências singulares, além de compor o processo de construção e expressão da identidade. E coletivo, pois cria um senso de pertencimento e integração ao deixar os ouvintes "mais confiantes com suas escolhas musicais e, ao mesmo tempo, permitir identificar-se com os outros" (Krastel et al., 2015, p. 5).

\footnotetext{
3 "The NPD Group: Music File Sharing Declined Significantly in 2012". NPD Group. Publicado em 26 de fevereiro de 2012. Disponível em: https://www.npd.com/wps/portal/npd/us/news/ press-releases/the-npd-group-music-file-sharing-declined-significantly-in-2012/.

4 "Streaming growth helps digital music revenues surpass physical sales". The Guardian. Publicado em 12 de abril de 2016. Disponível em: https://www.theguardian.com/ music/2016/apr/12/streaming-revenues-bring- big-boost-to-global-music-industry.
} 


\section{Descoberta musical na era digital}

A problemática específica da descoberta musical é um tema pouco teorizado nos estudos culturais e da música popular (Tepper; Hargittai, 2009; Nowak, 2016). Numa perspectiva sociológica em muito influenciada pela sociologia do gosto e da estratificação social de Bourdieu (2006), complementada com Hennion (2001, 2007), defende-se que a descoberta musical provém de uma correlação entre, por um lado, estruturas sociais e tecnológicas e, por outro, agência individual. Contudo, o processo e o momento da descoberta em si não são explorados de forma substantiva. É certo que é difícil retraçar com precisão a cadeia de elementos potenciadores da descoberta, pois se trata de um processo complexo e difuso. Ciente desta dificuldade, Nowak (2016) pretende sofisticar o olhar para a descoberta, perspectivando-a como um ato fenomenológico em que, para além da habitual preocupação com a mediação do contexto social e tecnológico situado, acrescenta uma dimensão afetiva. A procura pela experimentação do desconhecido, o ecletismo e conhecimento cultural são constantemente associados na literatura sociológica ao acúmulo de capital cultural, recurso que proporciona distinção social (Bourdieu, 2006; Peterson; Kern, 1996; Lahire, 2008), status (Tepper; Hargittai, 2009) e constitui parte do processo de construção identitária (DeNora, 2000).

Antes da internet, a descoberta exigia uma quantidade maior de recursos (tempo e dinheiro), e ficava limitada a lojas de discos, concertos ou revistas (Tepper; Hargittai, 2009). Estes agentes mediavam o processo de decisão de compra, agindo como gatekeepers - ou curadores. É difícil, no entanto, estimar o valor de um produto musical antes de experimentá-lo (Wikström, 2015). Ao contrário de filmes e livros, o ouvinte pode decidir se gosta ou não de uma música quase instantaneamente, ouvindo uma pequena amostra (Celma, 2008). No streaming, o livre acesso diminui a cautela, já que o ouvinte paga uma taxa única e tem direito ao consumo ilimitado em formato de subscrição (Fleischer, 2007) - de acordo com o catálogo disponível -, estimulando, no início, a experimentação (Dias et 
al., 2011). Esta nova lógica "alimenta a fantasia dos utilizadores da busca perfeita, da descoberta definitiva e da gratificação completa e instantânea através da imersão em milhões de commodities digitais intangíveis" (Burkart, 2014, p. 405). Além disso, como o acesso e o pagamento são realizados separadamente, o ouvinte tem "a sensação de que a música é gratuita" (Trefzger et al., 2015, p. 35).

A ampliação nas formas de acesso à música também está relacionada com uma tendência desvelada por muitos sociólogos da cultura que apontam para a emergência do omnívoro cultural, conceito popularizado por Peterson e Kern (1996) para designar um perfil de gostos diversificados, tanto da cultura de elite, como da cultura popular, misturando gêneros até então tidos como dissonantes (Lahire, 2008). Essa lógica "reflete uma mudança em nossos critérios de valor moral" (van Eijck, 2000, p. 219), já que ser tolerante a outros gêneros musicais é mais valorizado do que o esnobismo e senso de superioridade que dominava as elites socioculturais do passado. A busca pela variedade, hoje, é uma forma de diferenciar-se em meio à multidão. Warde et al. (2007, p. 145) lembram que há uma diferença, porém, entre gostar de tudo indiscriminadamente e estar aberto a novas experiências.

Apesar do livre acesso, um dos desafios para o ouvinte é lidar com o excesso de opções. Para McCourt e Zuberi (2016, p. 123), "a quantidade assombrosa de músicas disponíveis online proporciona maiores oportunidades de gatekeeping industrial e intervenção tecnológica" através da curadoria. O Spotify utiliza uma combinação de curadoria humana e algorítmica para sugerir e apresentar músicas compatíveis com os gostos e preferências musicais passadas dos utilizadores, funcionando como um fio condutor da experiência de consumo no presente.

Boa parte da curadoria passa a ser automatizada através de algoritmos sociotécnicos (Gillespie, 2014), combinando uma série de dados sobre o comportamento do usuário e tomando decisões de forma complexa e desconhecida. Para Morris (2015), inspirado na ideia do poder de filtragem e legitimação simbólica dos intermediários culturais de Bourdieu (2006), isto se 
chama de infomediação. Este é definido como as "entidades organizacionais que monitoram, coletam, processam e reembalam dados de uso cultural e técnico em uma infraestrutura informativa que molda a apresentação e a representação de bens culturais" (Morris, 2015, p. 452), o que "expande as capacidades das commodities digitais, auxiliando os utilizadores na descoberta de novos bens culturais ou oferecendo novas experiências de conteúdos familiares" (Morris, 2015, p. 453).

A mediação do conteúdo no Spotify, sob a ótica de Alzamora e Cortez (2014), é realizada por agentes de interface, responsáveis por funções mecânicas - como reconhecer padrões, hábitos e comportamentos dos utilizadores - e agentes humanos, responsáveis pela construção de significado através do acesso, consumo e compartilhamento das informações. Agentes de interface não são apenas extensões das atividades humanas, pois participam diretamente na experiência de consumo. Considerando as funções que ambos desempenham e seu grau de autonomia, a diferença entre os dois tipos de agentes está diminuindo, graças aos esforços da inteligência artificial.

Entretanto, por mais avançados que sejam os algoritmos, ainda não são capazes de alcançar o mesmo nível de sensibilidade da curadoria humana (McCourt; Zuberi, 2016). Mesmo que sejam aprimorados ao longo do tempo, algoritmos consideram apenas o comportamento passado do utilizador. A curadoria humana, por outro lado, é menos previsível e tende a ser mais ousada, sendo "capaz de agregar novas e inusitadas perspectivas à informação, oferecendo aos seus utilizadores a surpresa, o inesperado ou simplesmente aquilo que o utilizador nem imaginaria existir no mundo" (Corrêa; Bertocchi, 2012, p. 8).

Como forma de "colocar um toque pessoal na descoberta de músicas e enfatizar o valor de um serviço" (Glantz, 2016, p. 42), alguns serviços de streaming oferecem listas de reprodução criadas por especialistas, que fazem uma seleção de músicas com um humor, tema ou gênero em comum. $\mathrm{O}$ processo, no entanto, é uma atividade cara e pouco escalável, não sendo capaz de suportar o crescente volume de informações (Lindsay, 2016). Outra solução é combinar mais de um sistema de curadoria. O recurso 
Descobertas da Semana do Spotify fornece semanalmente uma playlist personalizada com 30 recomendações de músicas compatíveis com os gostos individualizados de cada ouvinte. Para fazer a seleção, o algoritmo combina dados do seu histórico de reprodução com informações da rede, sugerindo itens que outros utilizadores com gostos semelhantes consomem. Ao incorporar a curadoria humana no sistema, a recomendação se torna mais precisa, já que itens agrupados sinalizam uma forte correlação entre eles (Liu et al., 2016).

\section{A cauda longa da música}

Antes do streaming, a disponibilidade da música era limitada a elementos como o espaço nas prateleiras das lojas e o tempo de transmissão no rádio, fazendo com que o mercado se concentrasse na tentativa de maximização da audiência dos produtos mais rentáveis: os hits (Hesmondhalgh, 2013). Na era digital não há limites físicos no armazenamento. O custo de arquivo, distribuição e acesso diminuiu drasticamente, favorecendo a migração de um mercado de massa para um mercado de nichos. Este fenômeno é graficamente representado pela cauda longa (Anderson, 2006). Os consumidores devem, no entanto, "dispor de maneiras para encontrar os nichos que atendem às suas necessidades e interesses particulares" (Anderson, 2006, p. 51). Recursos de curadoria como mecanismos de busca e recomendação são importantes, pois fazem a ligação entre oferta e demanda, tornando os itens mais acessíveis ao longo da cauda. É uma forma de desvendar itens que seriam difíceis de ser encontrados de outras formas, como demonstrado por Zhong et al. (2013) em um estudo sobre o Last.fm. Segundo a pesquisa, as músicas mais curadas do serviço eram pouco presentes nas listas das mais reproduzidas.

Se, por um lado, as tecnologias digitais ampliam o acesso ao conteúdo, por outro lado, a abundância pode dificultar a experiência de consumo. Por este motivo, os mecanismos de curadoria são "ferramentas importantes para auxiliar-nos a descobrir novas músicas e redescobrir velhos favoritos" 
(Morris, 2012, p. 860). A previsibilidade das descobertas é, no entanto, um dos principais problemas apontados pelos autores (Goldenzwaig, 2014; Kennedy, 2015; Morris, 2015; Santos et al., 2015; Lindsay, 2016), pois, na tentativa de oferecer uma experiência personalizada, os serviços acabam por aprisionar o utilizador em espaços-comuns, restritos aos conteúdos familiares ou já conhecidos, criando uma espécie de "bolha virtual" (Pariser, 2011; Lovink, 2011). Um tipo de censura em que o acesso não é proibido, mas direcionado, "desestimulando para o conhecimento do novo" e criando "uma rejeição pelo que é diferente" (Santos et al., 2015, p. 6).

Para Kennedy (2015, p. 1), os serviços de streaming criam uma falsa percepção de controle e liberdade, limitando as escolhas e "sabendo o que queremos ouvir melhor do que nós mesmos". Segundo o autor, a verdadeira exploração acontece quando as descobertas são feitas em um ambiente "não mediado por qualquer fator controlador que não seja a própria restrição de tempo do indivíduo" (Kennedy, 2015, p. 4). Ao automatizar determinados processos, o streaming torna o consumo uma experiência mais passiva. As redes $\mathrm{P} 2 \mathrm{P}$ (peer to peer), pelo contrário, exigiam uma participação ativa do utilizador para descobrir novas músicas, pois não havia qualquer tipo de categorização e era necessário o descarregamento dos arquivos antes de poder ouvi-los. As limitações que havia antes do streaming seriam, de certa forma, libertadoras.

Parte do problema pode ser explicado pela forma como o ser humano toma decisões. O paradoxo da escolha ocorre quando um maior número de opções leva a piores decisões e níveis de insatisfação maiores (Oulasvirta et al., 2009).

\section{Playlists e acervos musicais}

Em menos de duas décadas, a música perdeu parte da sua materialidade e foi convertida em um fluxo de dados, transformando-se em uma "metáfora para a onipresença musical e a inesgotável escolha" (Morris; Powers, 2015, p. 109). Com isso, perde-se também a experiência tátil e material associada 
à música, desde a embalagem até a troca do disco. Para McCourt (2005), a música digitalizada tem menor valor emocional se comparada à mídia física, uma vez que a materialidade e a escassez estão ligadas à percepção de valor da música (Marshall, 2014; Goldenzwaig, 2014).

A era digital da música é caracterizada pelo êxtase no consumo, ao mesmo tempo em que há uma nostalgia com relação às antigas experiências. Como forma de distinção cultural (Bourdieu, 2006) e por oposição ao consumo digital, assiste-se a um fenômeno de reemergência do vinil (Sarpong et al., 2015). Em 2016, as vendas dos discos de vinil atingiram o maior índice desde 1991, tendo uma alta de 53\% com relação ao ano anterior ${ }^{5}$. Sá (2009) lista algumas das qualidades do vinil, como a longa durabilidade (superior à do CDs), a experiência tátil ao manusear o disco, a autenticidade sonora, a arte dos encartes e a ligação simbólica com a figura do DJ, já que ainda é amplamente usado para mixagens. Segundo a autora, o uso da mídia física estabelece uma conexão temporal com pessoas, lugares e momentos.

A desmaterialização da música começou com o MP3, mas intensificouse com o streaming, já que o indivíduo perde o controle sobre os arquivos, fazendo com que todos indivíduos tenham acesso a um único acervo musical. Colecionar música na era do streaming perde, assim, parte do sentido tradicional. A escassez é importante no processo de coleção, pois agrega valor aos itens colecionados e alimenta o desejo do colecionador, que está sempre à procura de algo novo. O streaming transforma a escassez em abundância, já que qualquer música se torna imediatamente disponível (Marshall, 2014).

A ampla disponibilidade e o fácil acesso alimentam o desejo pelo imediato, de forma que a "fluidez torna-se mais valorizada do que a história" e "a rapidez transforma-se em um fetiche" (McCourt, 2005, p. 250). O streaming é um retrato do "mundo pós-escassez, uma democracia na qual tudo tem o mesmo valor e disponibilidade". A experiência é substituída

5 "Record sales: vinyl hits 25-year high". The Guardian. Publicado em 3 de janeiro de 2017. Disponível em: <http://www.theguardian.com/music/2017/jan/03/record-sales-vinyl-hits25-year-high-and-outstrips-streaming > 
por "uma nova forma de tédio, de tornar-se aborrecido com a música que você está ouvindo, repetidamente pulando para a próxima faixa porque ela pode ser melhor, só porque você pode" (Marshall, 2014, p. 69).

Mesmo com a maior fluidez e intangibilidade, a coleção ainda é importante parte da experiência de consumo musical. Morris (2015, p. 459) acredita que, "em uma era em que o conteúdo não é de fato escasso, mas feito para parecer escasso, a organização reina". Toda coleção sugere uma ordem, caso contrário é apenas um acúmulo (Marshall, 2014, p. 63). A ordenação e categorização são feitas de acordo com uma série de critérios definidos pelo colecionador (Elsner; Cardinal, 1994). As playlists substituem antigas formas de organização do repertório musical e permitem que o utilizador se envolva em diálogos com o conteúdo musical, fazendo com que o valor esteja no processo, e não em sua existência (McCourt, 2005). Segundo Hagen (2015, p. 639), as playlists podem ter função de arquivar, contar histórias ou simplesmente organizar o conteúdo.

A personalização é uma forma de compensar a falta de materialidade no ambiente digital. Por isso, serviços de streaming ainda mantêm "laços simbólicos com mídias antigas" (Glantz, 2016, p. 44) através da forma como o conteúdo é apresentado, ou através de recursos linguísticos, a fim de criar um ambiente mais familiar e menos impessoal. No Spotify, por exemplo, o utilizador pode criar "estações de rádio" com músicas semelhantes a um artista ou canção. As próprias playlists são derivadas dos mixtapes, compilações de músicas gravadas de forma amadora em fitas cassete, populares na década de 1980, e que posteriormente foram adaptadas ao CD e ao MP3.

Para Zhong et al. (2013), a curadoria acontece ou de forma estruturada ou não estruturada. Ela é não estruturada quando o utilizador apenas destaca ou acumula itens, sem categorizá-los. Na curadoria estruturada, o indivíduo agrupa e coleciona os itens, categorizando-os de acordo com seus critérios, contextualizando a experiência de consumo. As playlists "podem conter muito contexto, e só os humanos podem ser capazes de interpretá-lo" (Celma, 2008, p. 53). O estudo de Cunningham et al., 2006 
apud Celma, 2008 mostrou que apenas 25\% das listas são organizadas de acordo com informações de conteúdo (como artista ou gênero). A forma como os utilizadores categorizam e rotulam suas músicas são uma forma de expressão, pois "prescreve como os utilizadores acessam e experimentam suas músicas" (Morris, 2012, p. 859).

\section{Metodologia de pesquisa}

Este estudo valeu-se de uma triangulação de métodos, incluindo entrevistas que possibilitaram acesso a aspectos pessoais que não seriam visíveis através da simples observação, como opiniões, motivações e experiências. Neste caso, a construção de significados acontece na interação entre os participantes, ao invés de concentrar-se unicamente no entrevistado ou no entrevistador (Tracy, 2013).

Os participantes foram recrutados a partir de uma publicação no Facebook. Entre os interessados, foram selecionados 10 homens e 10 mulheres. Todos deveriam obedecer aos seguintes critérios amostrais: ser utilizador do Spotify (free ou premium), ter entre 22 e 32 anos de idade, e residir no Brasil. Indivíduos nesta faixa etária tiveram, inicialmente, contato com formas antigas de consumo musical, como o MP3 e as redes P2P, por isso são capazes de comparar sua experiência no streaming com as primeiras plataformas de distribuição digital. Para além disso, de acordo com a literatura, são os mais jovens os mais predispostos a um tipo de consumo cultural omnívoro (Warde et al., 2007; Wel et al., 2008; Lahire, 2008; van Eijck, 2000).

Cientes da relativa homogeneidade da amostra e de que aspectos como padrões de aceso, práticas e motivações são estruturados pelo curso de vida e por variáveis de contextualização social, sobretudo o grau de escolaridade (López-Sintas et al., 2014), seria interessante, num outro momento, alargar a amostra para abarcar uma maior heterogeneidade etária e social.

As entrevistas foram semiestruturadas, utilizando um roteiro prédeterminado de perguntas. A vantagem de ter uma menor rigidez na 
entrevista é a possibilidade de "manter uma mente mais aberta sobre os contornos do que se precisa ou quer saber, de forma a que os conceitos e teorias possam emergir dos dados" (Bryman, 2012, p. 12). Quaisquer desvios no roteiro não são um problema, pelo contrário, permitem uma maior flexibilidade na coleta das informações (Silverman, 2013).

Nove entrevistas foram realizadas pela internet, através de videoconferências, e 11 foram realizadas presencialmente, em um local combinado com o participante. A videoconferência fornece uma riqueza maior de recursos não verbais em comparação com chamadas de voz. Além disso, poupa em recursos e imprime maior flexibilidade, já que permite realizar entrevistas durante o horário de trabalho de alguns participantes, e evita longos deslocamentos até a cidade dos entrevistados. Como uma forma de tornar os dados "manuseáveis" (Bryman, 2012, p. 13) para a análise, as transcrições do áudio de trechos selecionados das entrevistas foram feitas em conjunto com anotações utilizando um software de código livre: o Transcribe.

Além das entrevistas, foram recolhidos dados de utilização dos entrevistados no Spotify durante um intervalo de 45 dias, de forma a complementar e validar a pesquisa. Para isso, foi utilizado o IFTTT (If This Than That), um serviço online que automatiza tarefas através da definição de regras e da integração de diversas aplicações. Foram coletadas informações ${ }^{\mathbf{6}}$ como: a) músicas salvas na coleção; b) músicas adicionadas a playlists e c) títulos das playlists.

Para que a coleta dos dados funcione, o utilizador precisa autorizar o acesso do IFTTT à sua conta do Spotify. Por isso, foi enviado a cada participante um documento com instruções detalhadas sobre o procedimento. O texto de consentimento informado deixava claros os objetivos da pesquisa e dava a garantia de privacidade ao participante. A autorização foi totalmente feita pelos utilizadores e nenhuma senha precisou ser compartilhada. Após concedido o acesso, o serviço registrou os dados de forma cronológica numa tabela armazenada no Google Drive.

${ }^{6}$ Devido a limitações na API do Spotify, estes são os únicos dados possíveis de se obter até o momento. 
Coletar dados de utilização através de um software é uma forma de analisar o comportamento dos utilizadores sem que eles se sintam diretamente observados. Este potencial distanciamento é importante, uma vez que a presença do pesquisador pode fazer com que o participante regule seu comportamento ao sentir-se vigiado (Foucault, 1999), interferindo na experiência de ouvir música, que, muitas vezes, é realizada sem concentração total ou em simultâneo com outras tarefas cotidianas.

\section{Análise e discussão}

\section{Cartografia da história da relação dos entrevistados com a música}

Os participantes revelam uma trajetória de consumo musical parecida ao longo da vida. O primeiro contato com a música foi através do rádio ou do $\mathrm{CD}$. O consumo era limitado, pois o preço dos discos era elevado, e os lançamentos demoravam a chegar. A fita cassete, apesar de mais antiga, ainda era usada para gravar músicas que tocavam nas rádios. Mais tarde, com acesso à internet, a grande maioria utilizava ferramentas como Kazaa, LimeWire, eMule, Ares e BitTorrent para descarregar músicas. No início, a velocidade da conexão era baixa, o que dificultava o consumo. B.B. lembra que sua primeira coleção de músicas tinha apenas seis arquivos, pois levava muito tempo para descarregá-las. Com o passar do tempo, o download ilegal ficou mais fácil e barato, e foi a principal fonte de consumo de todos os participantes durante a última década. Muitos tinham o hábito de gravar CDs com músicas para ouvir no carro ou em outro aparelho, fazendo uma seleção das músicas para não ultrapassar o limite de armazenamento. Alguns também tinham reprodutores de MP3, como o iPod ou similares.

O repertório, no entanto, ficava limitado ao que os participantes conheciam através do rádio, televisão, revistas ou recomendações de amigos. A descoberta sempre foi muito influenciada pela mídia de massa e acontecia acidentalmente. Poucos disseram ter conhecido novidades através da internet na época, pois ainda não era tão utilizada. 
O YouTube foi e ainda é muito utilizado por todos, e na maioria dos casos significou o primeiro contato com o streaming. Antes do Spotify ser lançado, o YouTube já dava sugestões de vídeos relacionados, o que levava a descoberta de novas músicas. A plataforma, no entanto, nunca foi totalmente adequada ao consumo de músicas. F.P. reclama que, no celular, ao minimizar o aplicativo ou desligar a tela, a música para de tocar. Alguns entrevistados também utilizaram o Superplayer, um serviço de streaming brasileiro lançado em 2013, que tinha estações de rádio personalizadas, organizadas por uma equipe de curadores, e classificadas de acordo com gênero, ocasião ou humor, similares às playlists do Spotify. Não permitia escolher as músicas individualmente, sob demanda, apenas pular para as próximas da lista. O serviço alcançou um número expressivo de utilizadores antes de o Spotify chegar ao Brasil, em 2014.

Hoje, todos utilizam o Spotify como forma primária de consumo de música. A maior parte migrou rapidamente para o serviço, sem dificuldades. A interface gráfica do programa na versão desktop remete a softwares já familiares aos utilizadores, como iTunes e Windows Media Player. A possibilidade de login utilizando o perfil pessoal do Facebook também facilitou a entrada na plataforma, eliminando a necessidade de um novo cadastro.

Ao todo, 17 dos 20 participantes assinam a conta Premium, muito além da atual média global (que é de 50\% de utilizadores pagantes), muitos graças ao plano Familiar, que permite compartilhar a conta com parentes ou amigos. A interrupção da música pelos anúncios e a possibilidade de ouvir as faixas offline foram as principais motivações para a assinatura. Antes disso, existiam truques para contornar as limitações da conta gratuita. G.P. conta que criava listas com apenas cinco músicas, pois o aplicativo de celular só permitia pular de faixa quatro vezes.

Visto que o Spotify pode ser acessado de múltiplos dispositivos, a forma de utilização varia de acordo com a atividade e o dispositivo de acesso. O computador é mais usado para consumo durante o trabalho, quando permitido, e o uso do celular é mais frequente durante o trajeto para o trabalho e exercícios físicos. Para Sá (2009, p. 53) “há um conjunto de regras técnicas, 
socioculturais e políticas" ligadas à escuta em cada dispositivo, de modo que "parte do prazer (e também das limitações) da escuta musical advém das características materiais do aparelho". Sendo assim, pode-se dizer que não há um dispositivo preferido, apenas dispositivos mais adequados para diferentes situações e contextos. O consumo multidispositivos foi, inclusive, uma das principais transformações relatadas ocorrida graças ao uso do Spotify, pois facilita a sincronização da coleção entre vários aparelhos e acaba com a necessidade de transferir arquivos, como era feito na época do MP3.

Tal como indicado noutros estudos com entrevistas (Sinclair; Tinson, 2017), os termos facilidade e praticidade foram utilizados em todas as entrevistas para descrever a mudança nos hábitos de consumo pós-Spotify, referindo-se, principalmente, à automatização de diversos processos que, até então, exigiam tempo e esforço, como descarregar músicas, criar listas, organizar os arquivos e copiá-los para os dispositivos.

\section{Descoberta musical}

A facilidade e disponibilidade do acesso incentiva o consumo de uma variedade maior de músicas, mesmo entre os utilizadores menos ecléticos, que afirmam terem descoberto novas bandas e artistas com o Spotify. A descoberta varia, no entanto, de acordo com a disposição e necessidade de consumo de cada utilizador, e pode ocorrer de forma intencional ou ocasional.

A descoberta deliberada acontece de forma ativa, de acordo com a demanda, quando, por exemplo, o indivíduo cansa do seu repertório existente, ou por alguma necessidade específica. Por seu turno, a descoberta ocasional pode acontecer a qualquer hora e frequentemente surpreende o ouvinte. É provável que aconteça de forma passiva, ao escutar um artista desconhecido, uma playlist nova, ou até mesmo no dia a dia, ao ouvir na rua, no rádio ou na televisão. Este fenômeno é chamado de serendipidade (Merton; Barber, 2006), termo que descreve a sensação agradável de fazer descobertas bem-sucedidas por acidente ou acaso - que, na verdade, não 
acontecem por acaso, mas são condicionadas pelo algoritmo e por ações passadas do utilizador. Para M.B., este tipo de descoberta é mais valioso.

Eu tenho essa sensação permanente de querer muito conhecer coisas novas, mas você conhece umas coisas que não são tão boas. São nessas situações esporádicas que você não espera, tipo um amigo que tá ouvindo, ou tocando na rua, ou numa propaganda. É muito legal, mas é contar com a sorte (M.B.).

A descoberta é, portanto, um processo espontâneo e pouco premeditado, que depende do humor e da situação na qual o ouvinte se encontra. Há momentos em que a música é reproduzida como pano de fundo, durante outras atividades, como trabalho ou estudo, enquanto há momentos em que o ouvinte ativamente presta atenção no áudio. Diferente da televisão e do cinema, a música não exige atenção contínua, pois sua recepção acontece de forma omnidirecional, sem a necessidade de concentração (Anderson, 2011). Alguns preferem momentos de concentração para ouvir sons familiares e músicas que gostam, e deixam os momentos de distração para ouvir sons diferentes, aos quais não estão acostumados. Para Cannito (2009, p. 43), assim como "existe o interesse pela interação, pela concentração, pelo esforço [...] também existe o interesse pela passividade", principalmente na internet, onde a demanda por atenção e tomada de decisões é constante.

Apesar da comodidade em ouvir sons familiares, todos os participantes gostam de descobrir músicas novas, e todos foram capazes de citar ao menos um exemplo de descoberta recente através do Spotify. C.P. diz que a sensação de fazer uma descoberta que lhe agrada é comparável à de, antigamente, comprar um novo disco. Muitos contam que, ao descobrirem uma música de que gostam, escutam a música repetidamente, às vezes até "enjoar". Este é um comportamento comum, pois a música está ligada a recordações e memórias, provocando diversas sensações e lembranças (Anderson, 2011). Além de conteúdos novos, muitos relatam terem sido surpreendidos com redescobertas através do Spotify: músicas que ouviam 
há muito tempo atrás, mas que já haviam sido esquecidas ou que já não faziam mais parte do repertório.

Descobertas em outros meios, como televisão, rádio, cinema e concertos ainda acontecem, apesar de menos frequentes. O Shazam é bastante utilizado para identificação de músicas nestes casos. Indicações de amigos também são comuns, já que muitos utilizadores compartilham o link da música no Spotify através das redes sociais online ou aplicativos de mensagens. O YouTube segue sendo um significativo canal de consumo - pois tem músicas que não estão no catálogo do Spotify - e descoberta, graças aos recursos de sugestões.

Há inúmeras formas de alcançar um mesmo item no Spotify. A interface promove a intertextualidade e guia a experiência de consumo através de links que ligam um conteúdo ao outro. A barra de pesquisa geralmente é o ponto de partida, sendo a forma mais rápida e direta de chegar ao que procura. As playlists, apresentadas na tela inicial logo no primeiro acesso, são bastante utilizadas quando o utilizador não tem um objetivo tão específico além de ouvir música. A tela apresenta sugestões de listas de acordo com o horário, dia da semana ou dispositivo. Outros recursos, como os mecanismos de recomendação, estações de rádio e listas de conteúdos relacionados são ferramentas complementares que auxiliam no processo de descoberta, mas que nem sempre são utilizadas. A barra lateral, que mostra a atividade de outros utilizadores, também é uma fonte de descoberta e socialização, já que muitos dizem acompanhar o que os amigos ouvem, clicando frequentemente sobre o item que está sendo reproduzido por curiosidade. Além das Descobertas da Semana, o Daily Mix foi citado por alguns participantes, que dizem gostar do recurso, pois combina sugestões de músicas desconhecidas com músicas do repertório do utilizador. É atualizado diariamente e agrupado em mixes, de acordo com o gênero ou estilo musical.

É difícil julgar, no entanto, a qualidade das recomendações feitas por algoritmos, devido à falta de transparência dos seus critérios. Algumas sugestões são facilmente justificáveis, enquanto outras, incoerentes. Além 
disso, cada recurso tem objetivos diferentes, e podem ser mais ou menos adequados, dependendo do objetivo e expectativa de cada indivíduo. As Descobertas da Semana contêm sugestões mais ousadas e menos previsíveis, já o Daily Mix inclui músicas que o ouvinte já ouviu e conhece para criar um contexto mais familiar às sugestões apresentadas.

Um dos problemas identificados é a incapacidade do algoritmo em distinguir o consumo habitual do esporádico. Em situações e contextos de consumo coletivo, como em festas ou com os amigos, muitas das músicas reproduzidas são desvios de comportamento, geralmente para agradar outras pessoas ou simplesmente pela mudança de contexto. O algoritmo, no entanto, usa estas informações para determinar sugestões no futuro para um contexto de consumo individualizado, o que acaba desagradando.

Outro problema do Spotify, que afeta principalmente utilizadores novos, é a recomendação de itens muito previsíveis ou já conhecidos do passado. Na computação, este problema é chamado de cold start, e acontece quando o sistema não tem informações suficientes, por isso dá sugestões com menos precisão. Um novo utilizador, ao ingressar no Spotify, já tem uma identidade musical formada ao longo da sua trajetória pessoal e carrega uma bagagem cultural construída ao longo do tempo pelas experiências de consumo desde a infância, atravessando diversas fases, épocas, gêneros e mídias. O sistema, no entanto, apenas tem acesso a comportamentos recentes do indivíduo, pois não há input dos gostos e preferências além da própria experiência de consumo através da interação, da reprodução e da curadoria, desenvolvida com o passar do tempo.

\section{Consumo através de playlists}

As playlists são claramente a principal forma de consumo utilizada pelos participantes. Metade do tempo no Spotify hoje é gasto ouvindo playlists ${ }^{7}$. São, em sua maioria, criadas pela equipe de curadores do Spotify, mas

7 "The secret hit-making power of the Spotify playlist" de David Pearce. Wired. Publicado em 3 de maio de 2017. Disponível em: <https://www.wired.com/2017/05/secret-hitmaking-power-spotify-playlist/> 
também por gravadoras, artistas, marcas, e outros utilizadores. São adequadas, principalmente, para momentos em que o ouvinte não sabe exatamente o que quer ouvir, ou quer apenas uma trilha de fundo para o que estiver fazendo.

É comum seguir as listas que mais agradam, para receber notificações quando novas músicas são adicionadas. As mais seguidas entre os participantes são do Spotify, não só por terem maior destaque, mas também por serem diversificadas e atualizadas com maior frequência, segundo alguns participantes. A imagem de capa, o número de seguidores, a frequência de atualização e a reputação dos autores são indicadores que influenciam na percepção sobre a playlist, já que estes atributos são frequentemente associados a uma maior credibilidade e autoridade (Schaeffer, 2012).

As playlists refletem gostos e preferências musicais de cada entrevistado. Alguns utilizadores têm uma identidade clara e definida, como os fãs de rock, enquanto outros transitam entre diversos estilos musicais, geralmente não muito longe do mainstream. Uma tendência entre os mais ecléticos é seguir playlists de músicas mais tocadas, como Pop Up, Top Brasil, Brasil Top 50 , enquanto os ouvintes com gostos mais delimitados preferem playlists criadas de acordo com gêneros, estilos ou artistas. R.M. acha que este comportamento está ligado ao tipo de música que o indivíduo consome, já que a música pop é mais passageira e se renova com frequência. L.P. diz que "os roqueiros, em geral, são muito fechados a outros estilos".

Os rankings (ou paradas) de músicas no Spotify são, inclusive, importantes fontes de descoberta. Alguns utilizadores, antes mesmo antes do Spotify, já tinham o costume de acompanhar as listas de músicas mais populares em sites como Billboard (que elabora o Hot 100, principal ranking dos Estados Unidos) e Vagalume (site brasileiro de letras de música, que mantém uma lista das mais buscadas). Para L.R. e L.B., esta é uma forma de ficar atualizado sobre os lançamentos e novidades. Para R.M., o desempenho das músicas nos rankings guia sua experiência musical, pois funciona como um termômetro, uma espécie de validação ou comprovação do seu consumo.

A presença de uma canção em uma playlist popular pode ser determinante para seu sucesso. Um exemplo bastante citado nas entrevistas 
foi a canção Hear Me Now, lançada em outubro de 2016 pelo DJ brasileiro Alok. Ela tornou-se a primeira música brasileira a ultrapassar 100 milhões de reproduções no Spotify. Antes de lançar o single, o artista tinha cerca de 1,6 milhões de ouvintes mensais (em sua maioria, brasileiros). Três meses após o lançamento, este número passou para 13,4 milhões, um aumento de $738 \%$, segundo dados do Spotify ${ }^{8}$. Aqui, dois fenômenos podem ser observados: 1) a música ultrapassou a barreira do mercado nacional, ganhando visibilidade global a partir de playlists e rankings internacionais do Spotify; e 2) a música impulsionou o número de reproduções de outras canções do artista, formando uma nova cauda longa. Em fevereiro de 2017, das cinco cidades que mais ouviam a música, apenas duas eram brasileiras.

O sucesso de dois dos principais singles do Hot 100 em 2017, Rockstar (Post Malone) e Bodak Yellow (Cardi B) - de artistas até então desconhecidos - foram atribuídos ao desempenho viral que tiveram no Spotify ${ }^{9}$. A playlist RapCaviar - hoje uma das mais populares, com cerca de 8 milhões de seguidores, recentemente recebeu uma equipe editorial própria, conteúdo em vídeo e uma turnê de concertos, como uma espécie de submarca do Spotify, sendo responsável por apresentar diversos artistas de hip hop antes desconhecidos ${ }^{\mathbf{1 0}}$.

Sendo assim, ao mesmo tempo em que as playlists favorecem a distribuição do consumo através da cauda (Anderson, 2006), o tamanho da cauda também cresce, pois há mais oferta, aumentando a concorrência. Por outro lado, a facilidade de acesso no Spotify pode impulsionar a demanda por outras músicas de um mesmo artista, fazendo com que o consumo não fique limitado apenas a um único hit. Muitos participantes dizem que, ao

${ }^{8}$ Publicação do perfil oficial do Spotify Brasil (@spotifybrasil) no Instagram, em 27 de março de 2017. Disponível em: <https://www.instagram.com/p/BSJ6CUth29N/>

9 "How Rap Ruled Popular Music In 2017, From Cardi B to Post Malone" de Dan Hyman. Billboard. Publicado em 21 dez. 2017. Disponível em: < https://www.billboard.com/articles/ events/year-in-music-2017/8077743/cardi-b-post-malone-rap-ruled-year-in-music-2017> 10 "Spotify's RapCaviar turned the playlist into a movement, now Viva Latino is next" de Micah Singleton. The Verge. Publicado em 14 de Novembro de 2017. Disponível em: <https://www.theverge.com/2017/11/14/16635562/spotify-viva-latino-rap-caviar-playlistmusic-video-redesign > 
descobrirem um novo artista, procuram saber mais sobre ele. Geralmente ouvem as músicas mais reproduzidas e, caso gostem, ouvem o álbum ou discografia.

Nenhum entrevistado acredita que a abundância de conteúdo no Spotify seja um problema. G.A. diz que "nunca dá mais que três cliques para achar o que quer escutar". J.H. diz que a "síndrome do Netflix" não acontece no Spotify, pois não gasta muito tempo decidindo o que ouvir. R.M. também faz uma comparação com o Netflix, e diz que a experiência de consumo no Spotify é totalmente diferente, já que o tempo investido na decisão é menor, e a música é mais substituível que o vídeo. Caso não encontre a música que buscava, há muitas outras opções que agradam da mesma forma.

Além disso, boa parte dos entrevistados afirma ter expandido seu repertório com o uso do Spotify, pois é uma forma de consumir músicas que não ouviriam de outras formas, caso tivessem de comprá-las ou descarregálas, ou simplesmente nunca as descobririam. Mesmo aqueles que continuam ouvindo os mesmos gêneros acreditam que o uso dos recursos de descoberta é uma questão de preferência. Estar dentro de uma bolha (Pariser, 2011), se é que elas existem, não é uma obrigação, mas sim uma opção, uma forma de filtrar o abundante conteúdo do meio digital, muitas vezes inevitável e necessária. Nem todos desejam se afastar da cabeça da cauda ou da zona de conforto dos estilos musicais que já conhecem. A limitação do consumo e a descoberta de novas músicas são, portanto, assumidas como escolhas pessoais.

Um estudo ${ }^{11}$ recente conduzido pelo próprio Spotify revela que a diversidade de consumo - medida pela quantidade de artistas ouvidos por cada utilizador em uma semana - aumentou $37 \%$ nos últimos três anos. No mesmo período, a média de horas de música reproduzida por semana aumentou $25 \%$. A empresa atribui o aumento aos próprios recursos editoriais e algorítmicos, que incentivam e fomentam a descoberta e a redescoberta de músicas.

11 "Listening Diversity Increases Nearly 40 Percent on Spotify" de David Erlandsson e Jomar Perez, Spotify Insights. Publicado em 2 de novembro de 2017. Disponível em: <https:// insights.spotify.com/us/2017/11/02/listening-diversity-spotify/> 
No entanto, em tempos de superacessibilidade, a abundância acentua o desconforto causado por uma eventual escassez. Apesar de raro, alguns entrevistados relatam que já se frustraram por não encontrar determinado conteúdo no Spotify. A expectativa que se cria é de que, uma vez que a disponibilidade de músicas é tão grande, tudo pode ser encontrado. Porém, por maior que seja o catálogo do Spotify, há um ponto de corte. A cauda é longa, mas nunca vai ser infinita. É possível que o verdadeiro underground continue fora da plataforma, uma vez que tem uma postura contrária à cultura de massa e procura, através da obscuridade, manter-se longe da atenção e da visibilidade do mainstream (Hesmondhalgh, 1998).

Além disso, numa lógica de competição econômica, existem os eventuais conflitos de contrato e direitos autorais que levam um conteúdo a ser removido do Spotify. Os dois exemplos mais citados nas entrevistas foram os álbuns Lemonade (Beyoncé), e 1989 (Taylor Swift). Beyoncé lançou seu álbum exclusivamente no Tidal, serviço de streaming concorrente, e Taylor Swift removeu todos seus álbuns do catálogo em novembro de 2014, antes de voltar ao Spotify em junho de 2017. A solução encontrada pela maioria dos participantes, nesses casos, é recorrer ao YouTube.

Além do acesso, o streaming facilita a organização do repertório dos utilizadores por eliminar o esforço com download, edição e transferência de arquivos, processo que muitos consideravam trabalhoso e cansativo, e que, muitas vezes, limitava o consumo. A prática do mixtape é antiga, mas a seleção das músicas, muitas vezes, acontecia por pura necessidade, pois o armazenamento era limitado, e navegar entre as músicas era mais difícil. Hoje, apesar da maior facilidade, nem todos têm o hábito de criar e editar playlists - pelo menos não com uma frequência regular - já que existe uma grande oferta de listas editadas pelo Spotify, o que economiza tempo e esforço. A coleção, no entanto, ainda acontece, mesmo que de forma simbólica, já que o utilizador não é mais dono do conteúdo, mas aos poucos vai montando um repertório pessoal através de uma rede de links, favoritos e itens salvos. 
Eu desisti de fazer playlists, tirando a de favoritos, porque eu descobri que sempre vai ter uma playlist já criada por alguém muito próxima do que eu quero, e mais atualizada, pois a pessoa que faz dedica mais tempo, aí eu não vou fazer concorrência com quem só faz isso (R.M.).

Em termos funcionais, as playlists são frequentemente utilizadas como repositório e extensão da memória, a fim de recordar e ter fácil acesso às músicas preferidas. De modo análogo, o estudo de Hagen e Lüders (2016) mostra que o uso de playlists é uma forma de consolidar o repertório musical, combatendo "a tendência de seus hábitos de consumo tornarem-se fragmentados e efêmeros" (2016, p. 6). J.H., por exemplo, gosta de ter algumas coleções prontas para ocasiões em que não quer perder tempo decidindo o que ouvir. F.P. tem uma playlist onde salva todas suas músicas favoritas, porém reclama da falta de organização, pois a lista já tem mais de 500 faixas.

Além de repositórios, playlists pessoais são eficientes na "constituição de reputação de conhecimento sobre o assunto" (Amaral, 2009, p. 149), sendo um importante meio de expressão ao refletir gostos, hábitos e preferências pessoais. Semelhante ao processo de folksonomia descrito por Amaral e Aquino (2008, p. 3), a organização por playlists agrupa canções com sonoridades definidas, além de "códigos, comportamentos, rituais, roupas, gestos, gírias" e outras subjetividades que os utilizadores atribuem a um conjunto específico de músicas. Na maioria das vezes, são visíveis publicamente, mas há utilizadores que optam por mantê-las privadas quando revelam características mais íntimas sobre suas identidades musicais. Os critérios mais utilizados para criação de playlists são: gênero, humor (as sensações que a música provoca) e ocasião (evento ou marco temporal). Alguns participantes também possuem playlists criadas com base em um local, como trabalho ou academia.

As playlists podem agrupar músicas, sem ordem específica, geralmente reproduzidas de forma aleatória, ou podem contar uma história, sendo criadas e reproduzidas com uma sequência pré-determinada. $B$. B. diz que "a ordem da playlist importa, pois é algo bastante pessoal e para cada música há sempre uma próxima". Graças à maior facilidade, as playlists são 
mais dinâmicas se comparadas à época do MP3, pois é mais fácil adicionar, excluir e reordenar as canções. Além disso, o uso do armazenamento é mais eficiente, pois uma mesma música pode estar em múltiplas listas, sem que ocupe o espaço de vários arquivos. A interface do computador é mais adequada para a finalidade, já que permite arrastar e selecionar diversos itens de uma vez só, por exemplo.

Nem todas as listas são constantemente atualizadas. Muitas são criadas para uma ocasião específica, e depois abandonadas. As listas, neste caso, servem como ganchos temporais (Hagen, 2015), remetendo a um momento ou época da vida do indivíduo. Por este motivo, alguns utilizadores guardam suas playlists mais antigas como recordações, mesmo que nunca mais sejam atualizadas.

A playlist acaba sendo um resumo do meu ano, das festas que fui, das pessoas que conheci, das viagens que fiz, por isso a maioria das minhas playlists no Spotify tem o ano, pois vou me lembrar de qual foi (G.P.).

\section{Desmaterialização da música}

A percepção de valor da música após streaming não diminuiu, e sim aumentou, de acordo com as entrevistas. O principal motivo é a facilidade de distribuição e acesso, pois a música, hoje, pode chegar a mais pessoas por um preço "justo", sendo uma forma de remunerar os artistas pelo trabalho e reduzir a pirataria. F.D. acha que a tecnologia tornou a produção da música mais independente e democrática, permitindo o lançamento da música sem gravadoras, valorizando mais o artista do que os intermediários. Ao mesmo tempo, C.P., L.P. e R.M. acreditam que este novo modelo de distribuição pode ter alterado o ciclo de lançamento de músicas, de forma que muitos artistas lançam singles seguidamente sem lançar nenhum álbum. Apesar de ser um paradigma antigo da indústria fonográfica, esta tendência pode sinalizar uma mudança na relação do ouvinte com a música, que vem sendo consumida de forma não linear. 
Eu sempre via o álbum como uma coisa completa: é a capa, é a mensagem da capa, é pegar o encarte e ver as letras. Hoje você para de olhar a música como um artista ou como um álbum e você começa a olhar a música como um fragmento, uma célula isolada [...] e tem surgido muitos artistas de uma música só por esse motivo (L.P.).

O uso de formatos físicos de distribuição de música é raro e geralmente limita-se às coleções. A embalagem e apresentação do disco ainda são muito apreciadas pelos colecionadores, e podem ser uma forma de resistir à efemeridade da música. Tal prática vai ao encontro do fenômeno emergente de "reencarnação" de uma tecnologia retrô, como o vinil (Sarpong et al., 2015). É possível que a efemeridade na música seja uma das consequências da abundância de conteúdo e da superacessibilidade, já que a maior oferta de artistas e canções faça com que o tempo e a atenção sejam fracionados, provocando nostalgia e a percepção de que tudo é passageiro.

Por fim, o streaming reforça o conceito do indivíduo omnívoro (Peterson; Kern, 1996), já que alguns participantes acreditam que o Spotify favorece a formação de ouvintes mais ecléticos e tolerantes a novos estilos musicais, principalmente por dar visibilidade a gêneros que antes ficavam restritos a nichos, e muitas vezes discriminados, como o funk e o sertanejo. O acesso favorece a mobilidade, tanto dos ouvintes como dos artistas, entre diversos gêneros, que agora podem transitar por diversos estilos musicais com menos esforço. Ao mesmo tempo em que há mais segmentação, a distinção entre gêneros e estilos fica cada vez mais fluída.

Por outro lado, os recursos sociais do Spotify aumentam a exposição dos utilizadores, por exibirem hábitos que antes eram privados. A maior transparência no consumo, segundo Hagen e Lüders (2016), aumenta o nível de consciência dos indivíduos com relação ao que ouvem e a quem os observa. Uma vez que gostos musicais estão vinculados ao capital cultural (Bourdieu, 2006) e à reputação do indivíduo, a partilha do consumo de certos gêneros preocupa alguns utilizadores, pelo preconceito que carregam, e como tal há uma preocupação estratégica na apresentação que se faz do eu online (Johnson; Ranzini, 2018). 


\section{Considerações finais}

A música é um instrumento social sensível às mudanças tecnológicas, reverberando e (re)produzindo transformações na sociedade. A experiência de consumo na era do streaming reflete a atual condição da informação na era digital: é abundante, fragmentada e não linear. A facilidade e o baixo custo de acesso favorecem o consumo diversificado e aberto a descobertas, incentivando a experimentação e a exploração de músicas desconhecidas. Ao mesmo tempo, a superacessibilidade do conteúdo e a natureza sob demanda do serviço estimulam o consumo espontâneo e fragmentado da música, já que é possível alternar rapidamente entre diversas canções, artistas ou gêneros musicais com pouquíssimo esforço.

O consumo da música é muito influenciado pelo contexto, humor e nível de concentração do indivíduo, por isso o comportamento de consumo depende do local onde o utilizador se encontra, do dispositivo que utiliza, da atividade que realiza, entre outros fatores contextuais e individuais. A própria classificação do conteúdo por humor no Spotify coloca em questão a tradicional categorização por gênero ou estilo musical. Há momentos de maior passividade, quando a música serve como pano de fundo, assim como há ocasiões em que o consumo é mais ativo, de forma que o ouvinte tenha mais interação e controle sobre a reprodução. Do ponto de vista sensorial, a música evoca diferentes sentimentos e lembranças. Do ponto de vista social, serve como um objeto de diferenciação cultural, mas igualmente de aproximação, e faz parte do processo de construção da identidade.

Práticas antigas socializadas ao longo do tempo são necessárias para compreender muitos dos hábitos atuais no streaming. O consumo desmaterializado já acontecia com o rádio, o mixtape surgiu na época da fita cassete, e a coleção de discos foi, por muito tempo, uma atividade comum. Muitos dos recursos do Spotify demonstram um lastro histórico ao remeter a estas atividades, como as estações de rádio e a criação de playlists. Algumas práticas de curadoria no meio digital são representações simbólicas de atividades que o utilizador já fazia antes do streaming. Sendo assim, seu 
uso não cria novas lógicas de consumo, mas sim adaptações e domesticações (Silverstone; Hirsch, 1992; Berker et al., 2006) de comportamentos já existentes anteriormente ao contexto digital.

Também é interessante notar como o consumo musical deixa, ao longo do tempo, a esfera pública, e se torna mais individualizado e móvel (Bull, 2005; Beer, 2008) através dos dispositivos portáteis, dos fones do ouvido e, recentemente, da personalização oferecida pelos serviços de streaming, de forma que cada experiência seja única ao utilizador, ajustada com base nos seus gostos e preferências. Ao mesmo tempo, o Spotify cria um contexto favorável ao compartilhamento da experiência musical, mesmo que de forma assíncrona, ao integrar recursos de redes sociais, permitindo a criação de playlists colaborativas e de páginas com perfil de consumo dos utilizadores.

As playlists são o principal fio condutor da experiência de consumo, de forma a que a apreciação da música siga um contexto específico, ao invés de concentrar-se no artista e no álbum. O gatekeeping - que antes era restrito às gravadoras, às lojas e aos meios de comunicação em massa - torna-se mais distribuído, já que são inúmeras as formas de descobrir músicas, permitindo que os próprios utilizadores sejam curadores através de listas e recomendações automáticas. Ao mesmo tempo, o predomínio e influência do conteúdo curado pela equipe do Spotify leva a crer que os ouvintes preferem, na maioria das vezes, a conveniência de consumir playlists editoradas - de preferência, por especialistas. Apesar de ser constantemente aprimorada, a curadoria algorítmica ainda não substitui a curadoria humana, devido à maior previsibilidade, imprecisão e falta de transparência.

Pedro Henrique Moschetta é Mestre em Comunicação, Cultura e Tecnologias da Informação pelo ISCTE do Instituto Universitário de Lisboa.

$\equiv$ pedromoschetta@gmail.com

Jorge Vieira é Doutor em Sociologia e Professor Auxiliar Convidado do Departamento de Sociologia da Escola de Sociologia e Políticas Públicas do ISCTE no Instituto Universitário de Lisboa.

$\Xi$ jorge_vieira@iscte-iul.pt 


\section{Referências}

1. ALLEN-ROBERTSON, James. Digital culture industry: a history of digital distribution. Londres: Palgrave Macmillan, 2013.

2. ALZAMORA, Geane; CORTEZ, Natália. Agenciamentos semióticos em ambientes de streaming de músicas: mente, aprendizado e continuidade. Galaxia, n. 28, p. 173-83, 2014.

3. AMARAL, Adriana; AQUINO, Maria Clara. Práticas de folksonomia e social tagging no Last.fm. In: Anais - Conference on Human Factors in Computing Systems. Florença, 2008, p. 1-4.

4. AMARAL, Adriana. Plataformas de música online: práticas de comunicação e consumo através dos perfis. Revista Contracampo, n. 20, p. 147-70, 2009.

5. ANDERSON, Chris. A cauda longa. Tradução de Afonso Celso da Cunha Serra. 5 ed. Rio de Janeiro: Elsevier Editora, 2006.

6. ANDERSON, Jay. Stream capture: returning control of digital music to the users. Harvard Journal of Law \& Technology, v. 25, n. 1, p. 160-77, 2011.

7. BEER, David. Mobile music, coded objects and everyday spaces. Mobilities, v. 5, n. 4, p. 469-84, nov. 2010.

8. BEER, David. The iconic interface and the veneer of simplicity: MP3 players and the reconfiguration of music collecting and reproduction practices in the digital age. Information, Communication \& Society, v. 11, n. 1, p. 71-88, fev. 2008.

9. BERKER, Thomas et al. Domestication of media and technology. Berkshire: Open University Press, 2006.

10. BOURDIEU, Pierre. A distinção: crítica social do julgamento. Porto Alegre: Zouk, 2006.

11. BRYMAN, Alan. Social research methods. 4 ed. Oxford: Oxford University Press, 2012.

12. BULL, Michael. No dead air! The iPod and the culture of mobile listening. Leisure Studies, v. 24, n. 4, p. 343-55, 1 out. 2005.

13. BURKART, Patrick. Music in the cloud and the digital sublime. Popular Music and Society, v. 37, n. 4, p. 393-407, 2014.

14. CANNITO, Newton Guimarães. A TV 1.5 - a televisão na era digital. Tese de Doutorado - Escola de Comunicações e Artes, Universidade de São Paulo, São Paulo, 2009.

15. CELMA, Òscar. Music recommendation and discovery in the long tail. Tese de Doutorado - Departament de Tecnologies de la Informació i les Comunicacions, Universitat Pompeu Fabra, Barcelona, 2008.

16. CORRÊA, Elizabeth Saad; BERTOCCHI, Daniela. O algoritmo curador - O papel do comunicador num cenário de curadoria algorítmica de informação. In: Anais do XXI Encontro Anual da Compós. Juiz de Fora, 2012, p. 1-15. 
17. CRESWELL, John W. Research design: qualitative, quantitative, and mixed methods approaches. 4aㅡ ed. Londres: SAGE Publications, 2014.

18. DENORA, Tia. Music in everyday life. Cambridge: Cambridge University Press, 2000.

19. DIAS, Morena M.; NOGUEIRA, Tiago; BISPO, Ronaldo. BLIP.fm e as novas formas de consumo musical online. In: Anais do XVI Congresso de Ciências da Comunicação na Região Sudeste. São Paulo, 2011, p. 1-12.

20. DU GAY, Paul et al. Doing cultural studies: the story of the Sony Walkman. Londres: SAGE Publications, 1997.

21. ELSNER, John; CARDINAL, Roger. Cultures of collecting. Londres: Reaktion Books, 1994.

22. FLEISCHER, Rasmus. If the song has no price, is it still a commodity? Rethinking the commodification of digital music. Culture Unbound Journal of Current Cultural Research, v. 9, n. 2, p. 146-162, 2017.

23. FLEISCHER, Rasmus; SNICKARS, Pelle. Discovering Spotify - A thematic introduction. Culture Unbound Journal of Current Cultural Research, v. 9, n. 2, p. 130-45, 2017.

24. FOUCAULT, Michel. Vigiar e punir. 20. ed. Petrópolis: Editora Vozes, 1999.

25. FUCHS, Christian. Social media: a critical introduction. Londres: Sage, 2017.

26. GALUSZKA, Patryk. Music Aggregators and Intermediation of the Digital Music Market. International Journal of Communication, v. 9, p. 1-20, 15 jan. 2015.

27. GILLESPIE, Tarleton. The relevance of algorithms. In: GILLESPIE, Tarleton; BOCZKOWSKI, Pablo J.; FOOT, Kirsten A. Media technologies: essays on communication, materiality, and society. Cambridge: MIT Press, 2014. p. 167-93.

28. GLANTZ, Mark. Internet radio adopts a human touch: a study of 12 streaming music services. Journal of Radio \& Audio Media, v. 23, n. 1, p. 36-49, 2016.

29. GOLDENZWAIG, Gregory D. Music consumption practices in the age of the cloud: listening to Russia. World of media: yearbook of Russian media and journalism studies, v. 2013, p. 39-59, 2014.

30. GOMES, Carolina et al. Spotify: streaming e as novas formas de consumo na era digital. In: Anais do XVII Congresso de Ciências da Comunicação na Região Nordeste. Natal, 2015, p. 1-11.

31. HAGEN, Anja Nylund; LÜDERS, Marika. Social streaming? Navigating music as personal and social. Convergence, v. 23, n. 6, p. 643-59, 2016.

32. HAGEN, Anja Nylund. The playlist experience: personal playlists in music streaming services. Popular Music and Society, v. 38, n. 5, p. 625-45, 2015.

33. HENNION, Antoine. Music lovers: taste as performance. Theory, Culture \& Society, v. 18, n. 5, p. 1-22, 1 out. 2001. 
34. HENNION, Antoine. Those things that hold us together: taste and sociology. Cultural Sociology, v. 1, n. 1, p. 97-114, 1 mar. 2007.

35. HESMONDHALGH, David. The British dance music industry: a case study of independent cultural production. The British Journal of Sociology, v. 49, n. 2, p. 234-51, 1998.

36. HESMONDHALGH, David. The cultural industries. 3 ed. Londres: SAGE Publications, 2013.

37. JOHNSON, Benjamin K.; RANZINI, Giulia. Click here to look clever: selfpresentation via selective sharing of music and film on social media. Computers in Human Behavior, v. 82, p. 148-58, 1 maio 2018.

38. KENNEDY, Walker. Music streaming services, programming culture, and the politics of listening. Honors Projects, v. 35, 2015.

39. KITCHIN, Rob. Thinking critically about and researching algorithms. Information, Communication \& Society, v. 20, n. 1, p. 14-29, 2017.

40. KOTARBA, Joseph A.; VANNINI, Phillip. Understanding society through popular music. 1 ed. Nova York: Routledge, 2009.

41. KRASTEL, Zachary; BASSELLIER, Geneviève; RAMAPRASAD, Jui. Enabling social connectedness: the role of social features on online music sites. In: Anais - Conference on Information Systems and Technology (CIST). Philadelphia, 2015, p. 1-24.

42. LAHIRE, Bernard. Indivíduo e misturas de géneros: dissonâncias culturais e distinção de si. Sociologia, Problemas e Práticas, n. 56, p. 11-36, 2008.

43. LINDSAY, Charles. An exploration into how the rise of curation within streaming services has impacted how music fans in the UK discover new music. Journal of Promotional Communications, v. 4, n. 1, p. 115-41, 2016.

44. LIU, Yuchen; CHECHIK, Dmitry; $\mathrm{CHO}$, Junghoo. Power of human curation in recommendation system. In: Anais - 25th International Conference Companion on World Wide Web - WWW'16 Companion. Nova York: ACM Press, 2016, p. 79-80.

45. LOBATO, Ramon; THOMAS, Julian. The informal media economy. Cambridge: Polity Press, 2015.

46. LÓPEZ-SINTAS, Jordi et al. Music Access Patterns: A Social Interpretation. Poetics, v. 46, p. 56-74, 2014.

47. LOVINK, Geert. Networks without a cause: a critique of social media. Cambridge: Polity Press, 2011.

48. MARSHALL, Lee. W(h)ither Now? Music collecting in the age of the cloud. In: MARSHALL, Lee; LAING, Dave. Popular music matters: essays in honour of Simon Frith. Abingdon: Routledge, 2014, p. 61-72.

49. MCCOURT, Tom; ZUBERI, Nabeel. Music and discovery. Popular Communication, v. 14, n. 3, p. 123-26, 2016. 
50. MCCOURT, Tom. Collecting music in the digital realm. Popular Music and Society, v. 28, n. 2, p. 249-52, 2005.

51. MERTON, Robert K.; BARBER, Elinor. The travels and adventures of serendipity: a study in Sociological Semantics and the Sociology of Science. Nova Jersey: Princeton University Press, 2006.

52. MORRIS, Jeremy W.; POWERS, Devon. Control, curation and musical experience in streaming music services. Creative Industries Journal, v. 8, n. 2, p. 106-23, 2015.

53. MORRIS, Jeremy W. Curation by code: infomediaries and the data mining of taste. European Journal of Cultural Studies, v. 18, n. 4-5, p. 446-63, 2015.

54. MORRIS, Jeremy W. Making music behave: metadata and the digital music commodity. New Media \& Society, v. 14, n. 5, p. 850-66, 2012.

55. NOWAK, Raphael. When is a discovery? The affective dimensions of discovery in music consumption. Popular Communication, v. 14, n. 3, p. 137-45, 22 jul. 2016.

56. OULASVIRTA, Antti; HUKKINEN, Janne P.; SCHWARTZ, Barry. When more is less: the paradox of choice in search engine use. In: Anais - 32nd Annual ACM SIGIR Conference. Boston, 2009, p. 516-23.

57. PARISER, Eli. O filtro invisível: o que a internet está escondendo de você. Tradução de Diego Alfaro. 1 ed. Rio de Janeiro: Zahar, 2012.

58. PETERSON, Richard A.; KERN, Roger M. Changing highbrow taste: from snob to omnivore. American Sociological Review, v. 61, n. 5, p. 900-7, 1996.

59. ROSENBAUM, Steven. Curation nation: how to win in a world where consumers are creators. Nova York: McGraw Hill, 2011.

60. SÁ, Simone P. de. O CD morreu? Viva o Vinil! In: PERPÉTUO, Irineu F., SILVEIRA, Sérgio A. da. O futuro da música depois da morte do CD. São Paulo: Momento Editorial, 2009, p. 49-73.

61. SANTOS, Mylena C. G.; RAMOS, Rebecca C.; RIOS, José Riverson A. C. Os "filtros-bolhas" da internet nos aplicativos de música online. In: Anais do XXXVIII Congresso Brasileiro de Ciências da Comunicação. Rio de Janeiro, 2015, p. 1-15.

62. SARPONG, David; DONG, Shi; APPIAH, Gloria. "Vinyl Never Say Die": The re-incarnation, adoption and diffusion of retro-technologies. Technological Forecasting and Social Change, v. 103, p. 109-18, 2015.

63. SCHAEFFER, Mark W. Return on influence: the revolutionary power of klout, social scoring, and influence marketing. 1 ed. New York: McGraw Hill, 2012.

64. SCHWARZ, Jonas A. Online file sharing: innovations in media consumption. Nova York: Routledge, 2014.

65. SILVERMAN, David. Doing qualitative research: a practical handbook. $4^{\mathrm{a}}$ ed. Londres: SAGE Publications, 2013. 
66. SILVERSTONE, Roger; HIRSCH, Eric. Consuming technologies: media and information in domestic spaces. Londres: Routledge, 1992.

67. SINCLAIR, Gary; TINSON, Julie. Psychological ownership and music streaming consumption. Journal of Business Research, v. 71, p. 1-9, 2017.

68. TEPPER, Steven J.; HARGITTAI, Eszter. Pathways to music exploration in a digital age. Poetics, v. 37, n. 3, p. 227-49, 2009.

69. TRACY, Sarah J. Qualitative research methods - collecting evidence, crafting analysis, communicating impact. 1a ed. Nova Jersey: John Wiley \& Sons, 2013.

70. TREFZGER, Timm et al. Streaming killed the download star! The business model of streaming services revolutionizes music distribution. Journal of Organizational Advancement, Strategy and Institutional Studies, v. 7, n. 1, p. 29-39, 2015.

71. VAN EIJCK, Koen. Richard A. Peterson and the culture of consumption. Poetics, v. 28, n. 2-3, p. 207-24, 2000.

72. WARDE, Alan; WRIGHT, David; GAYO, Modesto. Understanding cultural omnivorousness: or, the myth of the cultural omnivore. Cultural Sociology, v. 1, n. 2, p. 143-64, 2007.

73. WEL, Frits Van et al. Youth cultural styles: from snob to pop? Young, v. 16, n. 3, p. 325-40, ago. 2008.

74. WIKSTRÖM, Patrik. The music industry: music in the cloud. 2 ed. Cambridge: Polity Press, 2013.

75. WIKSTRÖM, Patrik. Will algorithmic playlist curation be the end of music stardom? Journal of Business Anthropology, v. 4, n. 2, p. 278-84, 2015.

76. ZHONG, Changtao et al. Sharing the loves: understanding the how and why of online content curation. In: Anais - The 7th International AAAI Conference on Weblogs and Social Media. Boston, 2013. 